\title{
On Fourier Transforms of Wavelet Packets
}

\author{
K. Ahmad, R. Kumar and L. Debnath
}

Abstract. This paper deals with the Fourier transform $\widehat{\omega}_{n}$ of wavelet packets $\omega_{n} \in L^{2}(\mathbb{R})$ relative to the scaling function $\varphi=\omega_{0}$. Included there are proofs of the following statements:

(i) $\widehat{\omega}_{n}(0)=0$ for all $n \in \mathbb{N}$.

(ii) $\widehat{\omega}_{n}(4 n k \pi)=0$ for all $k \in \mathbb{Z}, n=2^{j}$ for some $j \in \mathbb{N}_{0}$, provided $|\widehat{\varphi}|,\left|m_{0}\right|$ are continuous.

(iii) $\left|\widehat{\omega}_{n}(\xi)\right|^{2}=\sum_{s=0}^{2^{r}-1}\left|\widehat{\omega}_{2^{r} n+s}\left(2^{r} \xi\right)\right|^{2}$ for $r \in \mathbb{N}$.

(iv) $\sum_{j=1}^{\infty} \sum_{s=0}^{2^{r}-1} \sum_{k \in \mathbb{Z}}\left|\widehat{\omega}_{n}\left(2^{j+r}(\xi+2 k \pi)\right)\right|^{2}=1$ for a.a. $\xi \in \mathbb{R}$ where $r=1,2, \ldots, j$.

Moreover, several theorems including a result on quadrature mirror filter are proved by using the Fourier transform of wavelet packets.

Keywords: Wavelet packets, multi-resolution analysis, Fourier transform, quadrature mirror filter

AMS subject classification: 42C15, 41A30, 39B99

\section{Introduction}

The concsept of wavelets refers to a family of functions of the form (see Daubechies [4])

$$
\psi_{a, b}(x)=|a|^{-\frac{1}{2}} \psi\left(\frac{x-a}{a}\right)
$$

normalized by the factor $|a|^{-\frac{1}{2}}$ where $\psi \in L^{2}(\mathbb{R})$ and $a, b \in \mathbb{R}$ with $a \neq 0$ (see Debnath and Mikusinski [5]). Thus, $\psi_{a, b}$ is constructed from translation and dilation of a single function $\psi$, called mother wavelet. Physically, the mother wavelet appears as a local oscillation (or wave) on which most of the energy of the oscillation is concentrated in the frequency (or wave number) domain due to the Heisenberg uncertainty principle. The scaling (or dilation) parameter $a$ controls the width and the rate of oscillation and intuitively can be thought of controlling the frequency of the wavelets $\psi_{a, b}$. The translation parameter $b$ simply moves the wavelet throughout the domain.

If the dilation and translation parameters $a$ and $b$ are chosen such that $a=2^{m}$ and $b=n 2^{m}$ where $m, n \in \mathbb{N}$, then there exists a wavelet $\psi$ such that the family of funtions $\left\{\psi_{m, n}\right\}$,

$$
\psi_{m, n}(x)=2^{-\frac{m}{2}} \psi\left(2^{-m} x-n\right)
$$

K. Ahmad and R. Kumar: Dept. Math., Jamia Millia Islama, New Delhi - 110025, India L. Debnath: University of Central Florida, Dept. Math., Orlando, Florida 32816, USA ldebnath@pegasus.cc.ucf.edu; khalil@jmi.ernet.in 
constitute an orthonormal basis of the space of functions or signals in $L^{2}(\mathbb{R})$ which have finite energy (see Daubechies [4], and Debnath and Mikusinski [5]). Thus, the two discrete parameters $m$ and $n$ can be varied for the analysis of local features of a given function. These two degrees of freedom $m$ and $n$ have the natural ability to describe features at a variety of scales by adjusting $m$ and at any location by changing $n$. On the other hand, in a Fourier basis, by contrast, the basis functions represent a one-parametric family $\left(e^{i x}\right)^{n}$, indexed by the frequency parameter $n$, and hence, one can effectively analyze global, periodic and smooth features by varying this frequency parameter $n$. Consequently, a Fourier basis is, however, less effective for the analysis of located oscillations or structure.

If $\psi \in L^{2}(\mathbb{R})$ and $\psi_{a, b}$ is given by (1.1), then the integral transformation $W_{\psi}$ defined on $L^{2}(\mathbb{R})$ by

$$
\mathcal{W}_{\psi}[f](a, b)=\left(f, \psi_{a, b}\right)=\int_{-\infty}^{\infty} f(x) \overline{\psi_{a, b}(x)} d x
$$

is called a continuous wavelet transform of $f$.

Coifman at al. [2] introduced the concept of wavelet packets. The wavelet transform is generalized to produce a library of orthonormal basis of modulated wavelet packets, where each basis comes with a fast transform. By generalyzing the method of multiresolution decomposition, it is possible to construct orthonormal bases for $L^{2}(\mathbb{R}$ ) (see Daubechies [4]). Discrete wavelet packets have been thoroughly studied by Wickerhauser [11] who has also developed computer programs and implemented them. The wavelet packets allow more flexibility in adapting the bases to the frequency contents of a signal and it is easy to develop a fast wavelet packet transform. The power of wavelet packet lies on the fact that we have much more freedom in deciding which basis function can represent the given function. The best basis selection criteria and application to image processing can be found in $[10,11]$.

Several authors including Daubechies [3], Chui [1] , Mallat [7, 8] and Meyer [9] have studied Fourier transforms of wavelets and scaling functions. On the other hand, Coifman et al. [2], Wickerhauser [11] and Hernandez and Weiss [6] have obtained some results on Fourier transforms of wavelet packets.

The main objective of this paper is to study the Fourier transform of wavelet packets in order to generalize some results due to authors listed in the preceding paragraph. This is followed by a result on quadrature mirror filter based on the Fourier transform of wavelet packets.

\section{Notations and terminology}

We let $\mathbb{Z}$ and $\mathbb{R}$ denote the set of integers and real numbers, respectively. The inner product of two functions $f, g \in L^{2}(\mathbb{R})$ is denoted by $\langle f, g\rangle$ and is defined (see Debnath and Mikusinski [5]) by $\langle f, g\rangle=\int_{-\infty}^{\infty} f(x) \overline{g(x)} d x$. The norm of $f \in L^{2}(\mathbb{R})$ is denoted by $\|f\|$. The Fourier transform of any function $f \in L^{2}(\mathbb{R})$ is denoted by $\hat{f}$ and is defined as usual by $\hat{f}(\xi)=\int_{-\infty}^{\infty} f(x) e^{-i \xi x} d x$. The set $\ell^{2}(\mathbb{Z})$ is the vector space of absolutno square-summable sequences. 
Definition 2.1. A multi-resolution analysis consists of a sequence of closed subspaces $\left\{V_{j}\right\}_{j \in \mathbb{Z}}$ of $L^{2}(\mathbb{R})$ satisfying the following properties:

(i) $V_{j} \subset V_{j+1}$ for all $j \in \mathbb{Z}$.

(ii) $\overline{\cup_{j \in \mathbb{Z}} V_{j}}=L^{2}(\mathbb{R})$ and $\cap_{j \in \mathbb{Z}} V_{j}=\{0\}$.

(iii) For $f \in L^{2}(\mathbb{R}), f(x) \in V_{j}$ if and only if $f(2 x) \in V_{j+1}$ for all $j \in \mathbb{Z}$.

(iv) For some $\varphi \in V_{0},\{\varphi(\cdot-k)\}_{k \in \mathbb{Z}}$ is an orthonormal basis for $V_{0}$.

(v) There exists an isomorphism $I: V_{0} \rightarrow \ell^{2}(\mathbb{Z})$.

The function $\varphi$ whose existence is implied by condition (iv) is called scaling function of the given multi-resolution analysis. For every $f \in V_{0}$, there exists a unique sequence $\left\{a_{k}\right\}_{k \in \mathbb{Z}} \in \ell^{2}(\mathbb{Z})$ such that

$$
f(x)=\sum_{k \in \mathbb{Z}} a_{k} \varphi(x-k) .
$$

We assume

$$
\varphi_{j, k}(x)=2^{\frac{j}{2}} \varphi\left(2^{j} x-k\right) .
$$

Since $\varphi_{0, k}(x)=\varphi(x-k)$, in view of condition (iv) $\varphi_{0, k} \in V_{0}$ follows for all $k \in \mathbb{Z}$. Moreover, if $j \in \mathbb{Z}$, condition (iii) implies that $\left\{\varphi_{j, k}\right\}_{k \in \mathbb{Z}}$ is an orthonormal basis for $V_{j}$.

Let $W_{0}$ be the orthonormal complement of $V_{0}$ in $V_{1}$, that is $V_{1}=V_{0} \oplus W_{0}$. Then if we dilate the elements of $W_{0}$ by $2^{j}$, we obtain a closed subspace $W_{j}$ of $V_{j+1}$ as

$$
V_{j+1}=V_{j} \oplus W_{j} \quad(j \in \mathbb{Z})
$$

We consider $V_{0}=W_{-1} \oplus V_{-1}$ and observe that $\frac{1}{\sqrt{2}} \varphi(\dot{\overline{2}}) \in V_{-1} \subset V_{0}$. By condition (iv) we can express this function in terms of the basis $\{\varphi(\cdot-k)\}_{k \in \mathbb{Z}}$ to obtain

$$
\varphi\left(\frac{x}{2}\right)=\sqrt{2} \sum_{k \in \mathbb{Z}} h_{k} \varphi(x-k)
$$

where $h_{k}=\frac{1}{\sqrt{2}} \int_{\mathbb{R}} \varphi\left(\frac{x}{2}\right) \overline{\varphi(x-k)} d x$, the convergence of the series is in $L^{2}(\mathbb{R})$ and $\sum_{k \in \mathbb{Z}}\left|h_{k}\right|^{2}<\infty$. We take the Fourier transform of (2.1) to obtain

$$
\widehat{\varphi}(2 \xi)=m_{0}(\xi) \widehat{\varphi}(\xi)
$$

where

$$
m_{0}(\xi)=\frac{1}{\sqrt{2}} \sum_{k \in \mathbb{Z}} h_{k} e^{-i \xi k}
$$

This gives the result

$$
\widehat{\varphi}(\xi)=\left\{\prod_{n=1}^{j} m_{0}\left(2^{-n} \xi\right)\right\} \widehat{\varphi}\left(2^{-j} \xi\right) .
$$

If $j \rightarrow \infty$ and $\widehat{\varphi}(0)=1$, then $\widehat{\varphi}(\xi)=\prod_{n=1}^{\infty} m_{0}\left(2^{-n} \xi\right)$. It follows from $(2.2)$ that

$$
m_{0}(0)=1
$$


The sequence $\left\{h_{k}\right\}$ and its Fourier series $m_{0}(\xi)$ need to satisfy specific constraints that are related to the properties of the function $\varphi$ :

(a) If $\varphi \in L^{2}(\mathbb{R})$, then $\sum_{k \in \mathbb{Z}} h_{k}=\sqrt{2}$.

(b) $\varphi$ is compactly supported in $[p, q]$ if and only if $h_{k}=0$ for $k<p$ or $k>q$.

(c) When the constraints (a) and (b) are satisfied, the orthogonality of $\{\varphi(\cdot-k)\}_{k \in \mathbb{Z}}$ is ensured if and only if

$$
\left|m_{0}(\xi)\right|^{2}+\left|m_{0}(\xi+\pi)\right|^{2}=1
$$

and there exists a compact set $K$, congruent to $[-\pi, \pi]$ modulo $2 \pi$, such that $m_{0}\left(2^{-k \xi}\right) \neq 0$ for all $\xi \in K$ and $k>0$.

We then define

$$
m_{1}(\xi)=-e^{i \xi} \overline{m_{0}(\xi+\pi)}
$$

and

$$
\widehat{\psi}(\xi)=m_{1}\left(\frac{\xi}{2}\right) \widehat{\varphi}\left(\frac{\xi}{2}\right) \quad \text { where } \psi(x)=\sqrt{2} \sum_{k \in \mathbb{Z}} g_{k} \varphi(2 x-k)
$$

with $g_{k}=(-1)^{k} \bar{h}_{1-k}$. It follows from $(2.4)-(2.6)$ that

$$
m_{1}(0)=0 \quad \text { and } \quad m_{1}(\xi)=\frac{1}{\sqrt{2}} \sum_{k \in \mathbb{Z}} g_{k} e^{-i \xi k} .
$$

Now we consider a function $\psi \in W_{0}$ such that $\{\psi(\cdot-k)\}_{k \in \mathbb{Z}}$ is an orthonormal basis for $W_{0}$. If this is the case, then $\left\{2^{\frac{j}{2}} \psi\left(2^{j} \cdot-k\right)\right\}_{k \in \mathbb{Z}}$ is an orthonormal basis for $W_{j}$ for all $j \in \mathbb{Z}$ due to condition (iv) in Definition 2.1 and the definition of $W_{j}$. Hence $\left\{\psi_{j, k}\right\}_{j, k \in \mathbb{Z}}$ is an orthonormal basis for $L^{2}(\mathbb{R})$, which means that $\psi$ is an orthonormal wavelet on $\mathbb{R}$.

Now we state few lemmas which will be used in the proofs of theorems in Section 3.

Lemma 2.1. Let $\psi \in L^{2}(\mathbb{R})$ be an wavelet constructed from the scaling function $\varphi$ such that $|\widehat{\varphi}|$ and $\left|m_{0}\right|$ are continuous. Then $\widehat{\psi}(4 k \pi)=0$ for all $k \in \mathbb{Z}$.

Lemma 2.2 (see Mallat $[8]$ ). Let $\varphi \in L^{2}(\mathbb{R})$ be a scaling function. Then $\{\varphi(\cdot-$ $k)\}_{k \in \mathbb{Z}}$ is an orthonormal basis of $V_{0}$ if and only if $\sum_{k \in \mathbb{Z}}|\widehat{\varphi}(\xi+2 k \pi)|^{2}=1$ for a.a. $\xi \in \mathbb{R}$.

Lemma 2.3 (see Hernandez and Weiss $[6]$ ). Let $\psi \in L^{2}(\mathbb{R})$ be a wavelet associated with the scaling function $\varphi$. Then $|\widehat{\varphi}(\xi)|^{2}=\sum_{j=1}^{\infty}\left|\widehat{\psi}\left(2^{j} \xi\right)\right|^{2}$ for a.a. $\xi \in \mathbb{R}$.

Now we construct wavelet packets from multi-resolution analysis. In general, consider two sequences $\left\{a_{n}\right\},\left\{b_{n}\right\} \in \ell^{2}(\mathbb{Z})$ satisfying the requirements as stated in [11: p. 452]. If $H$ is a Hilbert space with orthonormal basis $\left\{e_{k}\right\}_{k \in \mathbb{Z}}$, then the sequences defined by

$$
\begin{aligned}
f_{2 n} & =\sqrt{2} \sum_{k \in \mathbb{Z}} a_{2 n-k} e_{k} \\
f_{2 n+1} & =\sqrt{2} \sum_{k \in \mathbb{Z}} b_{2 n-k} e_{k}
\end{aligned}
$$


are orthonormal bases of two orthonormal closed subspaces $H_{1}$ and $H_{0}$, respectively, such that $H=H_{1} \oplus H_{0}$. Using this "splitting trick" we now define the basic wavelet packets associated with a scaling function $\varphi$ as defined in multi-resolution analysis.

Let $\omega=\varphi$. The basic wavelet packets $\omega_{n} \quad\left(n \in \mathbb{N}_{0}\right)$ associated with the scaling function $\varphi$ are defined recursively by

$$
\begin{aligned}
\omega_{2 n}(x) & =\sqrt{2} \sum_{k \in \mathbb{Z}} h_{k} \omega_{n}(2 x-k) \\
\omega_{2 n+1}(x) & =\sqrt{2} \sum_{k \in \mathbb{Z}} g_{k} \omega_{n}(2 x-k)
\end{aligned}
$$

When $n=0$ in (2.8), we obtain

$$
\begin{aligned}
& \omega_{0}(x)=\sqrt{2} \sum_{k \in \mathbb{Z}} h_{k} \omega_{0}(2 x-k) \\
& \omega_{1}(x)=\sqrt{2} \sum_{k \in \mathbb{Z}} g_{k} \omega_{0}(2 x-k)
\end{aligned}
$$

It follows from $(2.9)_{2}$ that $\omega_{1}=\psi$. The Fourier transform of the functions given in (2.9) yields the relations

$$
\begin{array}{r}
\widehat{\omega}_{0}(\xi)=\prod_{j=1}^{\infty} m_{0}\left(2^{-j} \xi\right) \\
\widehat{\omega}_{1}(\xi)=m_{1}\left(\frac{\xi}{2}\right) \prod_{j=1}^{\infty} m_{0}\left(2^{-j} \xi\right) .
\end{array}
$$

More generally, equalities (2.9) are equivalent to the result

$$
\widehat{\omega}(\xi)=\prod_{j=1}^{\infty} m_{\varepsilon_{j}}\left(2^{-j} \xi\right)
$$

where $n=\sum_{j=1}^{\infty} \varepsilon_{j} 2^{j-1}$ with $\varepsilon=0$ or $\varepsilon=0$.

\section{Main result}

Several theorems describing the main results are proved in this section.

Theorem 3.1. If $\omega_{n} \in L^{2}(\mathbb{R})$ are wavelet packets related to the scaling function $\varphi=\omega_{0}$, then $\widehat{\omega}_{n}(0)=0$ for all $n \in \mathbb{N}$.

Proof. We recall (2.10). I tis clear that for $n>0$ the right-hand side of (2.10) must contain at leat one term of type $m_{1}\left(2^{-j} \xi\right)$ where $j$ may take any value from $\mathbb{N}$. Then the result follows by using (2.7) 
Theorem 3.2. If $\omega_{n} \in L^{2}(\mathbb{R})$ are wavelet packets associated with the scaling function $\varphi$, then $\widehat{\omega}_{n}(4 n k \pi)=0$ for all $k \in \mathbb{Z}$, where must be $n=2^{j}$ for $j \in \mathbb{N}_{0}$, provided $|\widehat{\varphi}|$ and $\left|m_{0}\right|$ are continuous.

Proof. From the definition of wavelet packets, we have (2.8). The use of the Fourier transform of $(2.8)_{1}$ yields

$$
\widehat{\omega}_{2 n}(\xi)=m_{0}\left(\frac{\xi}{2}\right) \widehat{\omega}_{n}\left(\frac{\xi}{2}\right) .
$$

Taking $n=2^{j}$ and $\xi=4 n k \pi=2^{j+2} k \pi$ we get $\widehat{\omega}(4 n k \pi)=\widehat{\omega}_{2^{j}}\left(2^{j+2} k \pi\right)$. Applying equality (3.1) recursively and using Lemma 2.1 we get

$$
\begin{aligned}
\widehat{\omega}_{n}(4 n k \pi) & =m_{0}\left(2^{j+1} k \pi\right) \widehat{\omega}_{2^{j}-1}\left(2^{j+1} k \pi\right) \\
& =m_{0}\left(2^{j+1} k \pi\right) m_{0}\left(2^{j} k \pi\right) \cdots m_{0}\left(2^{2} k \pi\right) \widehat{\omega}_{1}\left(2^{2} k \pi\right) \\
& =\left[\sum_{r=2}^{j+1} m_{0}\left(2^{r} k \pi\right)\right] \widehat{\psi}(4 k \pi) \quad\left(\text { since } \omega_{1}=\psi\right) \\
& =0
\end{aligned}
$$

and the statement is proved

Theorem 3.3. Suppose $h_{n}$ is an exact quadrature mirror filter satisfying the conditions

$$
\sum_{n \in \mathbb{Z}} h_{n-2 k} \bar{h}_{n-2 l}=\delta_{k l} \quad \text { and } \quad \sum_{n \in \mathbb{Z}} h_{n}=\sqrt{2} .
$$

Further, let $m_{0}$ and $m_{1}$ be defined by (2.3) and (2.6), respectively. Then

$$
\sum_{j \in \mathbb{Z}} e^{-i \xi j}\left\{\bar{h}_{j-2 k} m_{0}\left(\frac{\xi}{2}\right)+\bar{g}_{j-2 k} m_{1}\left(\frac{\xi}{2}\right)\right\}=\sqrt{2} e^{-\frac{i \xi k}{2}}
$$

where $g_{k}=(-1)^{k} \bar{h}_{1-k}$.

Proof. We define the operators $F_{0}, F_{1}: \ell^{2}(\mathbb{Z}) \rightarrow \ell^{2}(2 \mathbb{Z})$ by

$$
F_{0}\left\{s_{k}\right\}(j)=\sum_{k \in \mathbb{Z}} s_{k} \bar{h}_{k-2 j} \quad \text { and } \quad F_{1}\left\{s_{k}\right\}(j)=\sum_{k \in \mathbb{Z}} s_{k} \bar{g}_{k-2 j} .
$$

Their adjoints $F_{0}^{*}$ and $F_{1}^{*}$ defined by

$$
F_{0}^{*}\left\{s_{k}\right\}(j)=\sum_{j \in \mathbb{Z}} s_{j} \bar{h}_{k-2 j} \quad \text { and } \quad F_{1}^{*}\left\{s_{k}\right\}(j)=\sum_{j \in \mathbb{Z}} s_{j} \bar{g}_{k-2 j}
$$

are orthogonal projections on $\ell^{2}(\mathbb{Z})$. The operator $F$ defined by

$$
F\left(s_{k}\right)=F_{0}\left(s_{k}\right) \oplus F_{1}\left(s_{k}\right) \in \ell^{2}(\mathbb{Z}) \oplus \ell^{2}(2 \mathbb{Z})
$$

is orthogonal and

$$
F_{0}^{*} F_{0}+F_{1}^{*} F_{1}=I
$$


where $I$ is the identity operator. We can write (2.8) as

$$
\begin{aligned}
\omega_{2 n}(t-j) & =\sqrt{2} \sum_{k \in \mathbb{Z}} h_{k-2 j} \omega_{n}(2 t-k)=F_{0}\left\{\sqrt{2} \omega_{n}(2 t-k)\right\}(j) \\
\omega_{2 n+1}(t-j) & =\sqrt{2} \sum_{k \in \mathbb{Z}} g_{k-2 j} \omega_{n}(2 t-k)=F_{1}\left\{\sqrt{2} \omega_{n}(2 t-k)\right\}(j)
\end{aligned}
$$

where $\sqrt{2} \omega_{n}(2 t-k)$ is viewed as a sequence in $j$ for $(t, n)$ fixed. Using (3.2) we get

$$
\omega_{n}(2 t-k)=\frac{1}{\sqrt{2}} \sum_{k \in \mathbb{Z}} \bar{h}_{j-2 k} \omega_{2 n}(t-j)+\frac{1}{\sqrt{2}} \sum_{k \in \mathbb{Z}} \bar{g}_{j-2 k} \omega_{2 n+1}(t-j) .
$$

The Fourier transforms of (2.8) yield

$$
\begin{aligned}
\widehat{\omega}_{2 n}(\xi) & =m_{0}\left(\frac{\xi}{2}\right) \widehat{\omega}_{n}\left(\frac{\xi}{2}\right) \\
\widehat{\omega}_{2 n+1}(\xi) & =m_{1}\left(\frac{\xi}{2}\right) \widehat{\omega}_{n}\left(\frac{\xi}{2}\right) .
\end{aligned}
$$

Now the Fourier transform of (3.3) gives

$$
\begin{aligned}
\widehat{\omega}_{n}(2 \xi-k) & =\frac{1}{\sqrt{2}} \sum_{k \in \mathbb{Z}} \bar{h}_{j-2 k} \widehat{\omega}_{2 n}(\xi-j)+\frac{1}{\sqrt{2}} \sum_{k \in \mathbb{Z}} \bar{g}_{j-2 k} \widehat{\omega}_{2 n+1}(\xi-j) \\
\Rightarrow \frac{1}{2} \widehat{\omega}_{n}\left(\frac{\xi}{2}\right) e^{-\frac{i \xi k}{2}} & =\frac{1}{\sqrt{2}} \sum_{j \in \mathbb{Z}} \bar{h}_{j-2 k} \widehat{\omega}_{2 n}(\xi) e^{-i \xi j}+\frac{1}{\sqrt{2}} \sum_{j \in \mathbb{Z}} \bar{g}_{j-2 k} \widehat{\omega}_{2 n+1}(\xi) e^{-i \xi j} \\
\Rightarrow \frac{1}{2} \widehat{\omega}_{n}\left(\frac{\xi}{2}\right) e^{-\frac{i \xi k}{2}} & =\frac{1}{\sqrt{2}} \sum_{j \in \mathbb{Z}} e^{-i \xi j}\left\{\bar{h}_{j-2 k} \widehat{\omega}_{n}\left(\frac{\xi}{2}\right) m_{0}\left(\frac{\xi}{2}\right)+\bar{g}_{j-2 k} \widehat{\omega}_{n}\left(\frac{\xi}{2}\right) m_{1}\left(\frac{\xi}{2}\right)\right\} \\
\Rightarrow \widehat{\omega}_{n}\left(\frac{\xi}{2}\right) e^{-\frac{i \xi k}{2}} & =\sqrt{2} \sum_{j \in \mathbb{Z}} e^{-i \xi j}\left\{\bar{h}_{j-2 k} m_{0}\left(\frac{\xi}{2}\right)+\bar{g}_{j-2 k} m_{1}\left(\frac{\xi}{2}\right)\right\} \widehat{\omega}_{n}\left(\frac{\xi}{2}\right) \\
\Rightarrow e^{-\frac{i \xi k}{2}} & =\sqrt{2} \sum_{j \in \mathbb{Z}} e^{-i \xi j}\left\{\bar{h}_{j-2 k} m_{0}\left(\frac{\xi}{2}\right)+\bar{g}_{j-2 k} m_{1}\left(\frac{\xi}{2}\right)\right\}
\end{aligned}
$$

for a.a. $\xi \in \mathbb{R}$ and the statement is proved

Remark 1. If $k=0$, the equality of Theorem 3.3 reduces to $\sum_{j \in \mathbb{Z}} e^{-i \xi j}\left\{\bar{h}_{j} m_{0}\left(\frac{\xi}{2}\right)+\right.$ $\left.\bar{g}_{j} m_{1}\left(\frac{\xi}{2}\right)\right\}=\sqrt{2}$.

Remark 2. If $\xi=0$, the equality of Theorem 3.3 together with $m_{0}(0)=1$ and $m_{1}(0)=0$ reduces to $\sum_{j \in \mathbb{Z}} \bar{h}_{j-2 k}=\sqrt{2}$ which can be written as $\sum_{j \in \mathbb{Z}} \bar{h}_{j}=\sqrt{2}$.

Theorem 3.4. If $\omega_{n} \in L^{2}(\mathbb{R})$ are wavelet packets associated with the scaling function $\varphi=\omega_{0}$, then

$$
\left|\widehat{\omega}_{n}(\xi)\right|^{2}=\sum_{s=0}^{2^{r}-1}\left|\widehat{\omega}_{2^{r} n+s}\left(2^{r} \xi\right)\right|^{2}
$$


for $r=0,1,2,3$.

Proof. From the definition of wavelet packets we have (2.8). Also, the Fourier transforms of wavelet packets are given by (3.3). Using (2.5) - (2.6) in (3.4) gives

$$
\begin{aligned}
\left|\widehat{\omega}_{2 n}(2 \xi)\right|^{2}+\left|\widehat{\omega}_{2 n+1}(2 \xi)\right|^{2} & =\left|m_{0}(\xi) \widehat{\omega}_{n}(\xi)\right|^{2}+\left|m_{1}(\xi) \widehat{\omega}_{n}(\xi)\right|^{2} \\
& =\left\{\left|m_{0}(\xi)\right|^{2}+\left|e^{i \xi} m_{0}(\xi+\pi)\right|^{2}\right\}\left|\widehat{\omega}_{n}(\xi)\right|^{2} .
\end{aligned}
$$

That is, $\left|\widehat{\omega}_{2 n}(2 \xi)\right|^{2}+\left|\widehat{\omega}_{2 n+1}(2 \xi)\right|^{2}=\left|\widehat{\omega}_{n}(\xi)\right|^{2}$. A simple iteration of this result yields $\left|\widehat{\omega}_{n}(\xi)\right|^{2}=\sum_{s=0}^{2^{r}-1}\left|\widehat{\omega}_{2^{r} n+s}\left(2^{r} \xi\right)\right|^{2}$ and the statement is proved

Remark 3. If $n=0$, the equality of Theorem 3.4 reduces to

$$
\left|\widehat{\varphi}_{n}(\xi)\right|^{2}=\left|\widehat{\omega}_{0}(\xi)\right|^{2}=\sum_{s=0}^{2^{r}-1}\left|\widehat{\omega}_{s}\left(2^{r} \xi\right)\right|^{2} .
$$

Theorem 3.5. If $\omega_{n} \in L^{2}(\mathbb{R})$ are wavelet packets associated with the scaling function $\varphi=\omega_{0}$, then

$$
\sum_{k \in \mathbb{Z}} \sum_{s=0}^{2^{r}-1}\left|\widehat{\omega}_{s}\left(2^{r}(\xi+2 k \pi)\right)\right|^{2}=1
$$

for a.a. $\xi \in \mathbb{R}$ where $r \in \mathbb{N}_{0}$.

Proof. In view of $(3.5)$ we can write $|\widehat{\varphi}(\xi+2 k \pi)|^{2}=\left|\widehat{\omega}_{0}(\xi+2 k \pi)\right|^{2}$. By using Theorem 3.4, the right-hand side of this equality can be written as

$$
|\widehat{\varphi}(\xi+2 k \pi)|^{2}=\sum_{s=0}^{2^{r}-1}\left|\widehat{\omega}_{s}\left(2^{r}(\xi+2 k \pi)\right)\right|^{2}
$$

which implies

$$
\sum_{k \in \mathbb{Z}} \sum_{s=0}^{2^{r}-1}\left|\widehat{\omega}_{s}\left(2^{r}(\xi+2 k \pi)\right)\right|^{2}=\sum_{k \in \mathbb{Z}}|\widehat{\varphi}(\xi+2 k \pi)|^{2}=1
$$

by Lemma 2.2 and completes the proof

Theorem 3.6. If $\omega_{n} \in L^{2}(\mathbb{R})$ are wavelet packets associated with the scaling function $\varphi=\omega_{0}$, then

$$
\sum_{j \in \mathbb{Z}} \sum_{s=0}^{2^{r}-1} \sum_{k \in \mathbb{Z}}\left|\widehat{\omega}_{n}\left(2^{j+r}(\xi+2 k \pi)\right)\right|^{2}=1
$$

for a.a. $\xi \in \mathbb{R}$ where $r=1,2, \ldots, j$.

Proof. From Theorem 3.4 we have

$$
\left|\widehat{\omega}_{1}(\xi)\right|^{2}=\sum_{s=0}^{2^{r}-1}\left|\widehat{\omega}_{2^{r}+s}\left(2^{r} \xi\right)\right|^{2}=\sum_{n=2^{r}}^{2^{r+1}-1}\left|\widehat{\omega}_{n}\left(2^{r} \xi\right)\right|^{2}
$$


since $\omega_{1}=\psi$. Lemma 2.2 together with Lemma 2.3 gives

$$
\sum_{k \in \mathbb{Z}}|\widehat{\varphi}(\xi+2 k \pi)|^{2}=\sum_{j=1}^{\infty} \sum_{k \in \mathbb{Z}}\left|\widehat{\omega}_{1}\left(2^{j}(\xi+2 k \pi)\right)\right|^{2} .
$$

Therefore,

$$
\sum_{j=1}^{\infty} \sum_{k \in \mathbb{Z}}\left|\widehat{\omega}_{1}\left(2^{j}(\xi+2 k \pi)\right)\right|^{2}=1
$$

for a.a. $\xi \in \mathbb{R}$. From equalities (3.6) and (3.7) we obtain the equality in question where, clearly, $r$ ranges form 1 to $j$ since it is not possible to decompose a wavelet space $W_{j}$ greater than $j$ times

Theorem 3.7. For orthonormal wavelet packets $\omega_{n} \in L^{2}(\mathbb{R})$ the expression

$$
D(\xi)=\sum_{j=1}^{\infty} \sum_{s=2^{r}}^{2^{r+1}-1} \sum_{k \in \mathbb{Z}}\left|\widehat{\omega}_{n}\left(2^{j+r}(\xi+2 k \pi)\right)\right|^{2}
$$

is well defined and finite for a.a. $\xi \in \mathbb{R}$. Moreover, $\int_{I} D(\xi) d \xi=2 \pi$ for any interval $I$ of length $2 \pi$ in $\mathbb{R}$.

Proof. $D(\xi)$ is well defined and can not be infinite on a set of positive measure if the last part of the theorem is true. The second part will follow if we prove the result for $I=[0,2 \pi]$ since $D(\xi)$ is $2 \pi$-periodic. Now,

$$
\begin{aligned}
\int_{I} D(\xi) d \xi & =D(\xi)=\sum_{j=1}^{\infty} \sum_{s=2^{r}}^{2^{r+1}-1} \sum_{k \in \mathbb{Z}} \int_{0}^{2 \pi}\left|\widehat{\omega}_{n}\left(2^{j+r}(\xi+2 k \pi)\right)\right|^{2} d \xi \\
& =\sum_{j=1}^{\infty} \sum_{s=2^{r}}^{2^{r+1}-1} \sum_{k \in \mathbb{Z}} \int_{2 k \pi}^{2(k+1) \pi}\left|\widehat{\omega}_{n}\left(2^{j+r} \xi\right)\right|^{2} d \xi \\
& =2 \pi \sum_{j=1}^{\infty} \sum_{s=2^{r}}^{2^{r+1}-1} \int_{-\infty}^{\infty}\left|\widehat{\omega}_{n}\left(2^{j+r} \xi\right)\right|^{2} d \xi \\
& =2 \pi \sum_{j=1}^{\infty} \sum_{s=2^{r}}^{2^{r+1}-1} 2^{-j-r} \int_{-\infty}^{\infty}\left|\widehat{\omega}_{n}(\xi)\right|^{2} d \xi \\
& =2 \pi \sum_{j=1}^{\infty} 2^{-j-r} \sum_{n=2^{r}}^{2^{r+1}-1}\left\|\widehat{\omega}_{n}\right\|_{2}^{2} \\
& =2 \pi \sum_{j=1}^{\infty} 2^{-j-r} 2^{r}\left\|\widehat{\omega}_{n}\right\|_{2}^{2} \\
& =2 \pi\left\|\widehat{\omega}_{n}\right\|_{2}^{2} \\
& =2 \pi
\end{aligned}
$$

and the statement is proved 
Acknowledgement. Authors express grateful thanks to referees for their suggestions that help revise the entire manuscript.

\section{References}

[1] Chui, C. K.: An Introduction to Wavelets. New York: Acad. Press 1992.

[2] Coifman, R., Meyer, Y., Quake, S. and M. V. Wickerhauer: Signal Processing and Compression with Wavelet Packets. Techn. Report. Yale Univ. 1990.

[3] Daubechies, I.: Orthonormal bases of compactly supported wavelets. Comm. Pure Appl. Math. 41 (1988), 909 - 996.

[4] Daubechies, I.: Ten Lectures on Wavelets (CBS-NSF Regional Conf. Appl. Math.: Vol. 61). Philadelphia: SIAM 1992.

[5] Debanth, L. and P. Mikusinski: Introduction to Hilbert Spaces with Applications (2nd ed.). New York: Acad. Press 1999.

[6] Hernandez, E. and G. Weiss: A First Course on Wavelets. Boca Raton (USA): CRC Press 1996.

[7] Mallat, S.: Multiresolution Approximations and wavelet orthonormal bases of $L^{2}(\mathbb{R})$. Trans. Amer. Math. Soc. 315 (1989), 69 - 87.

[8] Mallat, S.: A theory for multiresolution signal decomposition: The wavelet representation. IEEE Trans. Pattern. Anal. Machine Intell. 11 (1989), 674 - 693.

[9] Meyer, Y.: Wavelets and Operators. Cambridge: Univ. Press 1992.

[10] Wickerhauer, M. V.: Acoustic signal procession with wavelet packets. In: Wavelets: A Tutorial in Theory and Applications (ed.: C. K. Chui). Boston: Acad. Press 1992, pp. $679-700$.

[11] Wickerhauer, M. V.: Adapted Wavelet Analysis from Theory to Software. Wellesley (USA): A. K. Peters Ltd.

Received 17.08.1999; in revised form 12.04.2001 